\title{
Epidemiology, species distribution, and predictive factors for mortality of candidemia in adult surgical patients
}

\author{
Wei Zhang ${ }^{1}$, Xingpeng Song ${ }^{2}$, Hao $\mathrm{Wu}^{1}$ and Rui Zheng ${ }^{\text {** }}$
}

\begin{abstract}
Background: We evaluated the epidemiology, clinical characteristics, microbiology, outcomes, and risk factors for mortality of candidemia in adult surgical patients in Shenyang from 2012 to 2018.

Methods: We designed a retrospective observational study of adult patients with candidemia in a teaching hospital including three hospital campuses. Data regarding clinical and demographic characteristics were collected from the patient's medical records.

Results: Of the 236 cases of candidemia, 172 (72.9\%) were identified in surgical patients, including 146 (84.9\%) general surgeries, 11 (6.4\%) urologic surgeries, 6 (3.5\%) thoracic surgeries, and others. Higher proportions of solid tumors, total parenteral nutrition, the presence of a urinary catheter, and the presence of a gastric tube were observed in surgical patients with candidemia versus non-surgical ones, whereas the percentages of hematological malignancy, diabetes mellitus, and renal replacement therapy were relatively lower in surgical patients. Renal failure, leukopenia, and thrombocytopenia were less common laboratory findings in surgical patients with candidemia than compared to non-surgical ones. Among surgical patients with candidemia, Candida parapsilosis was the predominant species (43\%), followed by C. albicans (33.7\%), C. glabrata (11\%), C. tropicalis (8.1\%), and others (4.1\%). Overall susceptibility, susceptible dose dependent or intermediate susceptibility, and resistance to fluconazole were detected in 73.3, 19.8, and 3.5\% Candida isolates from surgical patients, respectively, but no resistance to amphotericin B was observed. Overall, the 30-day mortality in surgical patients was 19.2\%. At multivariable analysis, independent risk factors for death in surgical patients with candidemia were ICU stay, thrombocytopenia, and C. albicans infection.
\end{abstract}

Conclusions: Surgical patients account for the majority of candidemia cases. Among patients with recent surgery, risk factors for species distribution, antifungal sensitivity patterns of Candida isolates causing candidemia, and independent risk factors for mortality should be evaluated and considered for a better outcome in the antifungal treatment.

Keywords: Candidemia, Surgery, Candida parapsilosis, Risk factor, Mortality

\footnotetext{
* Correspondence: zhengr@sj-hospital.org

${ }^{1}$ Department of Pulmonary and Critical Care Medicine, Shengjing Hospital of

China Medical University, No. 36 Sanhao Street, Heping District, Shenyang

110004, Liaoning, China

Full list of author information is available at the end of the article
}

(c) The Author(s). 2020 Open Access This article is licensed under a Creative Commons Attribution 4.0 International License, which permits use, sharing, adaptation, distribution and reproduction in any medium or format, as long as you give appropriate credit to the original author(s) and the source, provide a link to the Creative Commons licence, and indicate if changes were made. The images or other third party material in this article are included in the article's Creative Commons licence, unless indicated otherwise in a credit line to the material. If material is not included in the article's Creative Commons licence and your intended use is not permitted by statutory regulation or exceeds the permitted use, you will need to obtain permission directly from the copyright holder. To view a copy of this licence, visit http://creativecommons.org/licenses/by/4.0/ The Creative Commons Public Domain Dedication waiver (http://creativecommons.org/publicdomain/zero/1.0/) applies to the data made available in this article, unless otherwise stated in a credit line to the data. 


\section{Background}

Several studies have reported that Candida species account for up to $9-22 \%$ of all nosocomial bloodstream infections (BSIs) [1, 2], which implies high-mortality rates and prolonged hospitalization, as well as increased hospital costs [1, 3-5]. Prior surgery has been demonstrated to one of the main risk factors of candidemia [6,7]. Over the past decades, the proportion of surgical patients in candidemia cases has shown an increasing trend [8]. In recent years, it is reported that approximately $50-55 \%$ of patients with candidemia have undergone recent surgery prior to the development of candidemia [4, 5, 9]. The risk of occurrence of candidemia among patients who underwent a surgical procedure or had a central venous catheter (CVC) were 11 times higher than those who did not have a surgical procedure or a CVC [6]. The previous prospective multicenter study conducted in surgical intensive care units (ICUs) has identified several risk factors independently associated with the increased risk of candidemia, including prior surgery, acute renal failure, receipt of parenteral nutrition, and, for postsurgical patients, presence of a triple lumen catheter [6].

Some studies on candidemia have been performed in surgical and critically ill patients $[6,10]$ or surgery wards [11], however those focusing on candidemia in surgical patients are limited [12]. Therefore, this retrospective study was undertaken to evaluate the epidemiology, clinical and microbiologic characteristics, outcomes, and prognostic factors of candidemia in adult surgical patients in Shenyang.

\section{Patients and methods}

\section{Subjects and data collection}

A retrospective observational study on adult (age $\geq 14$ years old) hospitalized patients of candidemia was conducted from January 2012 to December 2018 at a tertiary grade A comprehensive hospital located in Shenyang, China. The setting is an over 6000-bed universityaffiliated teaching hospital including three hospital campuses.

Candidemia was defined as the isolation of Candida spp. from at least one blood culture in patients with clinical signs and symptoms of infection. For patients with multiple positive blood culture, only the first case of candidemia was included, and furthermore a new episode of candidemia was defined if more than 30 days. The onset of candidemia was defined as the date when the first positive blood culture was collected. One patient undergoing subtotal gastrectomy was excluded as the identification of Staphylococcus epidermidis and C. glabrata from the same blood culture were both considered contaminants. Besides those lost to follow-up within 30 days after the onset of candidaemia were excluded. Surgical patients refer to those undergoing any recent surgery. Recent surgery was defined as within 3 months of surgery from the onset of candidemia, while other predisposing factors, such as ICU stay, total parenteral nutrition (TPN), presence of CVC, mechanical ventilation, presence of urinary catheter, presence of gastric tube, were defined as occurring within 30 days before the onset of candidemia. Prior antibiotic exposure and prior antifungal exposure were defined as the administration of any antibiotics or antifungal drugs during the 30 days before the onset of candidemia. Antifungal therapy was considered as empirical if any antifungal therapy started within $24 \mathrm{~h}$ of the time the blood culture specimen was drawn but before the species identification, and as targeted after the species identification. Abdominal surgery refers to any operation involving organs including the stomach, small intestines, the colon or rectum, the gallbladder, the liver, pancreas, the spleen and the appendix. The abnormal laboratory results were defined as follows: renal failure (a creatinine clearance $<60 \mathrm{~mL} / \mathrm{min}$ ), anemia (a hemoglobin level of $<110 \mathrm{~g} / \mathrm{L}$ in women and $<130 \mathrm{~g} / \mathrm{L}$ in men), hypoalbuminemia (a serum albumin concentration $<30 \mathrm{~g} / \mathrm{L}$ ), hyponatremia (a serum sodium level $<135 \mathrm{mmol} / \mathrm{L}$ ), leukopenia (peripheral white blood cell count $<4 \times 10^{9}$ cells/L), and thrombocytopenia (platelet count $<100 \times 10^{9}$ cells $/ \mathrm{L}$ ).

Demographic and clinical data regarding basic characteristics, treatment, and clinical outcomes, laboratory data were collected from the electronic medical records during the study period. The study was approved by the Medical Ethics Committee in Shengjing Hospital of China Medical University (reference number 2019PS636K).

\section{Microbiological analysis}

The microbiological laboratory methods for blood culture, Candida species identification, and antifungal susceptibility testing were performed as previously described [13]. The antifungal susceptibilities of the antifungal agents were evaluated according to clinical breakpoints (CBPs) recommended by the Clinical Laboratory Standards Institute (CLSI) [14] or European Committee on Antimicrobial Susceptibility Testing (EUCAST) [15].

\section{Statistical analysis}

Data were analyzed with SPSS (IBM SPSS Statistics 21.0) statistical software. The Kolmogorov-Smirnov test was applied to evaluate the normal distribution of continuous data. Normally distributed continuous variables were expressed as mean \pm SEM (standard error of the mean) and compared using Student's $t$-test. Non-normally distributed data were reported as the median and the 25th75th percentiles and analyzed with Mann-Whitney $U$ test. Categorical variables were presented as number (\%) and compared between groups by the $X^{2}$ test or $X^{2}$ continuity correction test. Those variables with a $P$-value < 0.1 in the univariable analysis were entered into the 
multivariable logistic regression analysis model to determine independent risk factors for 30-day mortality, and the results were presented as odds ratios (OR) with their 95\% confidence intervals (CI) and $P$-values. A two-tailed $P$-value of $<0.05$ was considered statistically significant.

\section{Results}

During the study period 2012-2018, we identified a total of 236 episodes of candidemia occurring in 232 adult patients, of whom 172 patients with candidemia had undergone recent surgery with an incidence of $24.8 \mathrm{epi}$ sodes per 1000 patient-days. Figure 1 presents the annual incidence rates of candidemia in surgical patients using annual patient-days, which varied from 19.2 to 32 episodes per 1000 patient-days during the reported study periods. Overall, the downward trend in the annual incidence rates of candidemia in surgical patients from 2012 to 2018 could not be demonstrated as no statistical significance was found in the linear regression analysis in Fig. 1. $(P=0.34)$.

\section{Candida species distribution}

In the study overall, C. parapsilosis $(74,43.0 \%)$ was the leading causative organism of candidemia in surgical patients, followed by C. albicans (58, 33.7\%), C. glabrata (19, 11\%), C. tropicalis (14, 8.1\%), C. famata (3, 1.7\%), C. guilliermondii (2, 1.2\%), C. krusei (1, 0.6\%), and one only identified to Candida genus level (1, 0.6\%). The distribution of Candida spp. isolates from surgical patients with candidemia are outlined in Fig. 2. During the 7-year period, C. parapsilosis and C. albicans remained the first and second most frequently isolated species for each year from 2012 to 2018, respectively. And although $C$. glabrata remained the third most commonly isolated species, its percentage remarkably decreased from $20.8 \%$ (2012) to 8.3\% (2018) (Fig. 2A). The presence of C. parapsilosis in surgical patients was significantly higher than that in non-surgical patients $(43.0 \%$ vs. $23.4 \%, P=0.006)$, while the presence of $C$. tropicalis was lower $(8.1 \%$ vs. $20.3 \%, P=0.009$ ) (Table 1). Additionally, among surgical patients with candidemia, C. tropicalis isolates were more likely to occur in elderly patients $(13.3 \%$ vs. $4.1 \%$, $P=0.03)$ and in patients with diabetes mellitus (DM) (21.9\% vs. $5 \%, P=0.005$ ) (Fig. 2 B).

\section{Patient characteristics}

One hundred and seventy-two cases had received recent surgery prior to the onset of candidemia, including 146 (84.9\%) general surgeries, 11 (6.4\%) urologic surgeries, 2 (1.2\%) gynecological and obstetric surgeries, 6 (3.5\%) thoracic surgeries, $4(2.3 \%)$ neurosurgeries, and $3(1.7 \%)$ orthopedic surgeries. Of these, 119 (69.2\%) cases were

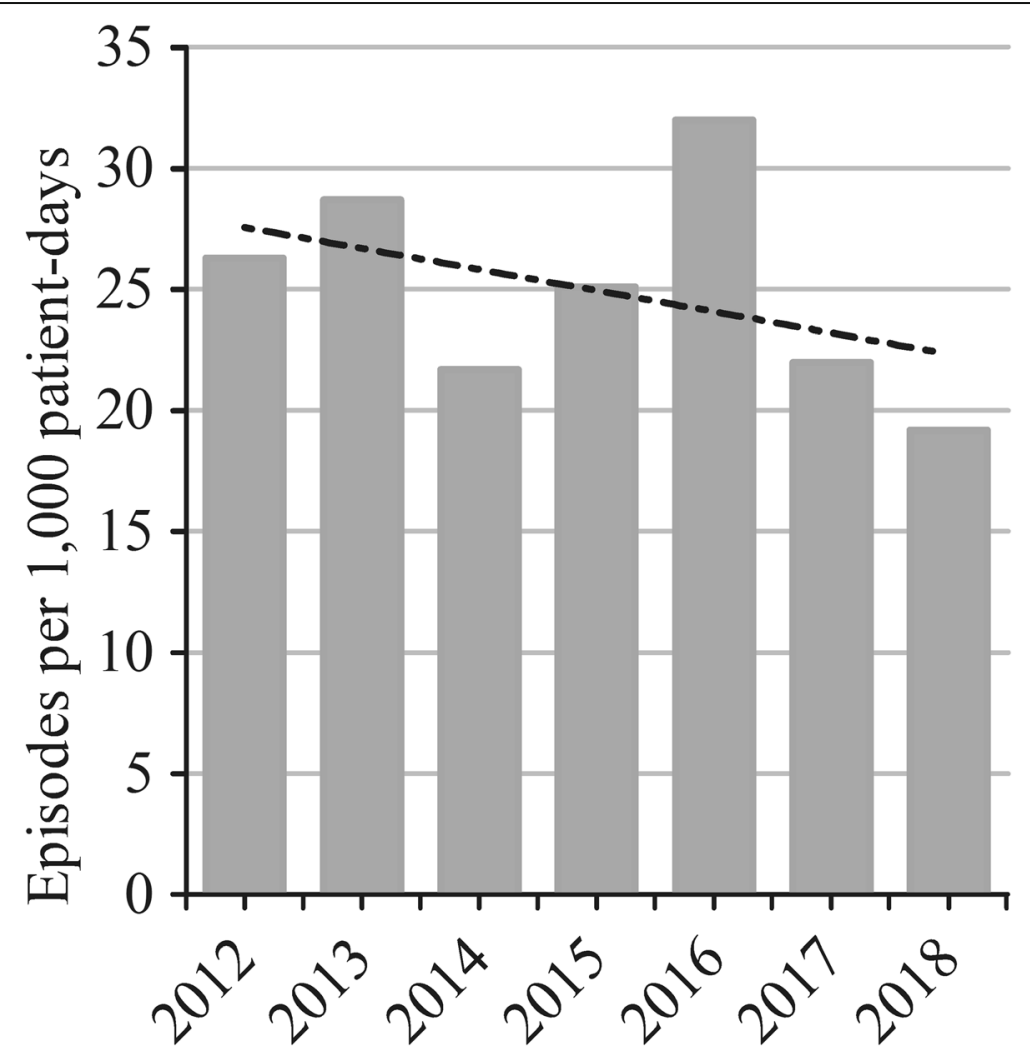

Fig. 1 Annual candidemia incidence density in surgical patients, 2012 to 2018 

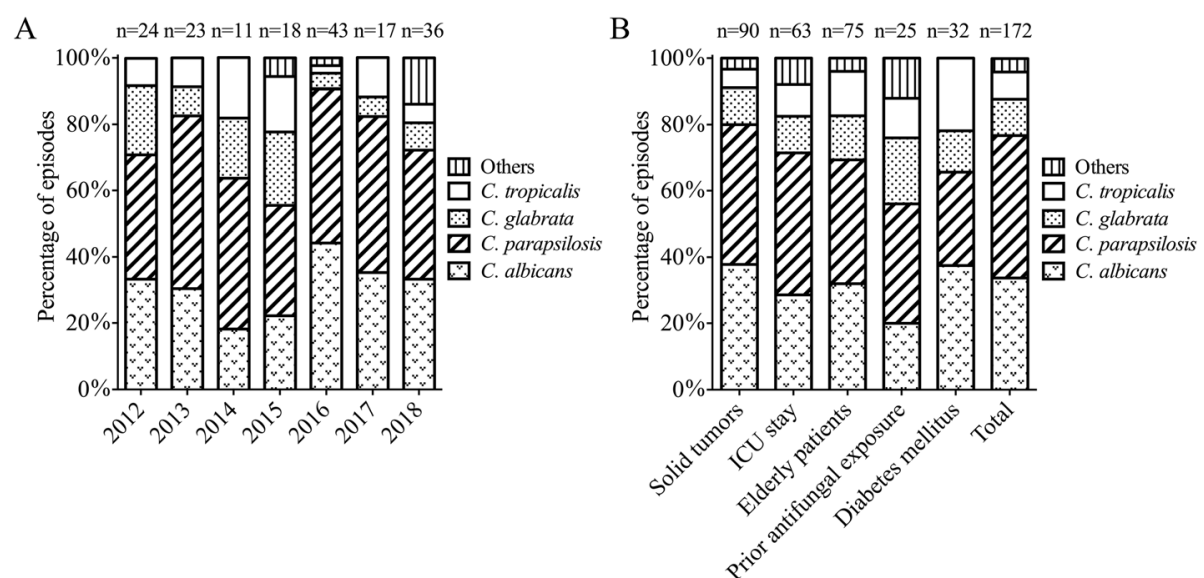

Fig. 2 Distribution of Candida species isolates from surgical patients with candidemia during the study period according to the time period (A) and underlying diseases or predisposing factors (B)

male, and 33 (19.2\%) had CVC-related candidemia. The comparison of demographics and clinical characteristics between surgical and non-surgical patients with candidemia is summarized in Table 1 . Of note, surgical patients were older (mean age: $62.5 \pm 14.2$ years vs. $55 \pm 21.9, P=0.002)$ and had longer length of hospital stay (LOS) (median LOS: 32 vs. 26 days, $P=0.02$ ) than those non-surgical patients. In addition, compared with non-surgical patients, the prevalence of solid tumors $(52.3 \%$ vs. $12.5 \%, P<0.001)$ was significantly higher in surgical patients, whereas the percentages of hematological malignancy $(0.6 \%$ vs. $23.4 \%$, $P<0.001)$ and DM $(18.6 \%$, vs. $37.5 \%, P=0.002)$ were lower. As expected, TPN (94.2\% vs. $40.6 \%, P<0.001)$, the presence of a urinary catheter $(73.8 \%$ vs. $32.8 \%$, $P<0.001)$, and the presence of a gastric tube $(76.7 \%$ vs. $35.9 \%, P<0.001$ ) were remarkably more frequent in surgical patients with candidemia versus nonsurgical patients. Moreover, a higher rate of previous antibiotic therapy was documented for surgical patients than for controls $(96.5 \%$ vs. $81.3 \%, P<0.001)$. Instead, the proportion of renal replacement therapy in surgical patients was lower when compared to non-surgical patients $(4.1 \%$ vs. $23.4 \%, P<0.001)$. However, the proportion of patients who had ICU stay or received the presence of a CVC and mechanical ventilation, and 30-day mortality was similar between the two groups. Regarding laboratory findings, renal failure $(18 \%$ vs. $40.6 \%, P<0.001)$, leukopenia $(11 \%$ vs. $29.7 \%, P=0.001)$, and thrombocytopenia $(14.0 \%$ vs. $37.5 \%, P<0.001)$ were less common in surgical patients with candidemia than in controls.

\section{Antifungal susceptibility testing}

Table 2 details antifungal susceptibility results of Candida spp. isolated from surgical patients with candidemia.
Among the 172 isolates tested, overall resistance to fluconazole was $3.5 \%$, susceptibility to fluconazole was $73.3 \%$, and susceptible dose dependent (SDD) or intermediate susceptibility to fluconazole was $19.8 \%$. For fluconazole, the highest susceptibility rate was identified in C. parapsilosis $(87.8 \%)$, followed by C. albicans (86.2\%) and C. tropicalis (78.6\%). Isolates of C. glabrata exhibited the greatest proportion of SDD to fluconazole (100\%), whereas the highest resistance rates were found in C. krusei $(100 \%)$ and C. tropicalis (14.3\%). Fluconazole susceptibilities of Candida isolates from candidemia with prior exposure to antifungal agents significantly decreased than those without ( $52 \%$ vs. $76.9 \%, P=0.009$ ). Overall, only $47.7 \%$ of isolates tested were susceptible to itraconazole. Furthermore, overall, $4.1 \%$ of isolates tested were resistant to voriconazole, and no resistance to amphotericin B was observed in our study.

\section{Antifungal therapy, outcomes and prognostic factors of candidemia in surgical patients}

Of 172 episodes of candidemia occurred in surgical patients, empiric antifungal therapy was administered in $115(66.9 \%)$ of the cases. The median duration of antifungal therapy was 9 (interquartile range 5-14) days in 145 surgical patients receiving any antifungal treatment. Among 27 (15.7\%) surgical patients who did not receive any antifungal treatment, 5 patients died before or on the day the blood culture results were obtained. Among the remaining 22 patients without any antifungal treatment, the fever improved after surgical procedures, including $\mathrm{CVC}$ removal in 14 patients, the drainage catheter removal in four patients, left pyelostomy in two patients, the peritoneal drainage in one patient, and the endoscopic nasal biliary drainage in one patient. Fluconazole was the most commonly administered initial agent (88.3\%), followed by micafungin (6.2\%) and caspofungin 
Table 1 Demographics and clinical characteristics of surgical and non-surgical patients with candidemia

\begin{tabular}{|c|c|c|c|}
\hline Characteristics & Surgical patients $(\boldsymbol{n}=172)$ & Non-surgical patients $(\boldsymbol{n}=64)$ & $\boldsymbol{P}$ value \\
\hline Age & $62.5 \pm 14.2$ & $55.0 \pm 21.9$ & 0.002 \\
\hline Age $\geq 65$ years & 75 (43.6\%) & $22(34.4 \%)$ & 0.2 \\
\hline Male sex & $119(69.2 \%)$ & $41(64.1 \%)$ & 0.45 \\
\hline \multicolumn{4}{|l|}{ Underlying diseases } \\
\hline Hematological malignancy & $1(0.6 \%)$ & $15(23.4 \%)$ & $<0.001$ \\
\hline Solid tumors & $90(52.3 \%)$ & $8(12.5 \%)$ & $<0.001$ \\
\hline Diabetes mellitus & $32(18.6 \%)$ & $24(37.5 \%)$ & 0.002 \\
\hline \multicolumn{4}{|l|}{ Predisposing factors } \\
\hline Renal replacement therapy & $7(4.1 \%)$ & $15(23.4 \%)$ & $<0.001$ \\
\hline Current and former smokers & $52(30.2 \%)$ & $18(28.1 \%)$ & 0.75 \\
\hline ICU stay & $63(36.6 \%)$ & $20(31.3 \%)$ & 0.44 \\
\hline Prior antibiotics exposure & $166(96.5 \%)$ & $52(81.3 \%)$ & $<0.001$ \\
\hline Prior antifungal exposure & $25(14.5 \%)$ & $12(18.8 \%)$ & 0.43 \\
\hline TPN & $162(94.2 \%)$ & $26(40.6 \%)$ & $<0.001$ \\
\hline Presence of CVC & $67(39.0 \%)$ & $26(40.6 \%)$ & 0.82 \\
\hline Mechanical ventilation & $20(11.6 \%)$ & $8(12.5 \%)$ & 0.85 \\
\hline Presence of urinary catheter & $127(73.8 \%)$ & $21(32.8 \%)$ & $<0.001$ \\
\hline Presence of gastric tube & $132(76.7 \%)$ & $23(35.9 \%)$ & $<0.001$ \\
\hline \multicolumn{4}{|l|}{ Laboratory findings } \\
\hline Renal failure & $31(18 \%)$ & $26(40.6 \%)$ & $<0.001$ \\
\hline Anemia & $144(83.7 \%)$ & 55 (85.9\%) & 0.68 \\
\hline Hypoalbuminemia & $71(41.3 \%)$ & $31(48.4 \%)$ & 0.32 \\
\hline Hyponatremia & $73(42.4 \%)$ & $18(28.1 \%)$ & 0.045 \\
\hline Leukopenia & $19(11 \%)$ & $19(29.7 \%)$ & 0.001 \\
\hline Thrombocytopenia & $24(14.0 \%)$ & $24(37.5 \%)$ & $<0.001$ \\
\hline C. albicans infection & $58(33.7 \%)$ & $25(39.1 \%)$ & 0.45 \\
\hline C. parapsilosis infection & $74(43.0 \%)$ & $15(23.4 \%)$ & 0.006 \\
\hline C. glabrata infection & 19 (11\%) & $10(15.6 \%)$ & 0.34 \\
\hline C. tropicalis infection & $14(8.1 \%)$ & $13(20.3 \%)$ & 0.009 \\
\hline Fluconazole susceptibility & $126(73.3 \%)$ & $44(68.8 \%)$ & 0.50 \\
\hline Empirical antifungal therapy & $115(66.9 \%)$ & $40(62.5 \%)$ & 0.53 \\
\hline No antifungal therapy & 27 (15.7\%) & $9(14.1 \%)$ & 0.76 \\
\hline \multicolumn{4}{|l|}{ Outcome } \\
\hline 30-day mortality & $33(19.2 \%)$ & $18(28.1 \%)$ & 0.14 \\
\hline LOS (days) & $32(22.3-47.8)$ & $26(15-47)$ & 0.02 \\
\hline
\end{tabular}

ICU, Intensive care unit; TPN, Total parenteral nutrition; CVC, central venous catheter; LOS, Length of hospital stay;

(5.5\%). The surgical patients with candidemia had lower 30-day mortality rates than those non-surgical patients (19.2\% vs. $28.1 \%)$, although no statistic differences presented $(P=0.14)$. Regarding the 30-day mortality rates in the different surgeries, excepting the rate of $0 \%$ in patients undergoing urologic surgeries, gynecological and obstetric surgeries, the 30-day mortality rate was highest in patients undergoing thoracic surgeries $(3,50 \%)$ or undergoing neurosurgeries (2, 50\%), followed by undergoing orthopedic surgeries $(1,33.3 \%)$, and undergoing general surgeries $(27,18.5 \%)$.

Within 30 days from the onset of candidemia, mortality occurred in 33 (19.2\%) surgical patients, 2 of whom or their guardians decided to quit therapy after a few days of antifungal therapy, 5 of whom have died before the positivity of blood cultures was reported, 3 of whom died after the antifungal treatment, and 23 of whom died during the antifungal treatment. Univariable analyses 
Table 2 In vitro antifungal susceptibility of Candida species isolated from surgical patients with candidemia

\begin{tabular}{|c|c|c|c|c|c|c|c|}
\hline \multirow{2}{*}{$\frac{\text { Species }}{(n=172)}$} & \multirow[t]{2}{*}{ Antifungal agent } & \multicolumn{3}{|l|}{ MIC $(\mu \mathrm{g} / \mathrm{ml})$} & \multicolumn{3}{|c|}{ No. (\%) of isolates by new $\mathrm{CBPs}^{\mathbf{a}}$} \\
\hline & & Ranges & $\mathrm{MIC}_{50}$ & $\mathrm{MIC}_{90}$ & $\mathrm{~S}$ & SDD / I & $\mathbf{R}$ \\
\hline \multirow[t]{4}{*}{ C. albicans $(n=58)$} & Fluconazole & $\leq 0.5-16$ & $\leq 1$ & 4 & $50(86.2 \%)$ & $6(10.3 \%)$ & $2(3.4 \%)$ \\
\hline & Voriconazole & $\leq 0.03-\leq 4$ & 0.06 & $\leq 1$ & 49 (84.5\%) & $3(5.2 \%)$ & $6(10.3 \%)$ \\
\hline & Itraconazole & $0.062-1$ & $\leq 0.125$ & 0.25 & NA & NA & NA \\
\hline & Amphotericin B & $\leq 0.25-1$ & $\leq 0.5$ & 0.5 & $58(100 \%)$ & 0 & 0 \\
\hline \multirow[t]{4}{*}{ C. parapsilosis $(n=74)$} & Fluconazole & $\leq 0.5-8$ & $\leq 1$ & $\leq 4$ & $65(87.8 \%)$ & $8(10.8 \%)$ & $1(1.4 \%)$ \\
\hline & Voriconazole & $\leq 0.03-0.25$ & $\leq 0.06$ & 0.125 & $72(97.3 \%)$ & $2(2.7 \%)$ & 0 \\
\hline & Itraconazole & $\leq 0.062-0.25$ & $\leq 0.125$ & 0.125 & $72(97.3 \%)$ & 0 & $2(2.7 \%)$ \\
\hline & Amphotericin B & $\leq 0.25-1$ & $\leq 0.5$ & 0.5 & $74(100 \%)$ & 0 & 0 \\
\hline \multirow[t]{4}{*}{ C. glabrata $(n=19)$} & Fluconazole & $\leq 1-16$ & 4 & 16 & 0 & 19 (100\%) & 0 \\
\hline & Voriconazole & $\leq 0.06-0.5$ & 0.125 & 0.25 & NA & NA & NA \\
\hline & Itraconazole & $\leq 0.125-1$ & 0.25 & 1 & NA & NA & NA \\
\hline & Amphotericin B & $\leq 0.25-1$ & $\leq 0.5$ & 0.5 & 19 (100\%) & 0 & 0 \\
\hline \multirow[t]{4}{*}{ C. tropicalis $(n=14)$} & Fluconazole & $\leq 1-32$ & 1 & 16 & $11(78.6 \%)$ & $1(7.1 \%)$ & $2(14.3 \%)$ \\
\hline & Voriconazole & $\leq 0.06-4$ & 0.06 & 0.5 & $12(85.7 \%)$ & $1(7.1 \%)$ & $1(7.1 \%)$ \\
\hline & Itraconazole & $\leq 0.125-8$ & 0.125 & 1 & $10(71.4 \%)$ & 0 & $4(28.6 \%)$ \\
\hline & Amphotericin B & $\leq 0.25-0.5$ & $\leq 0.5$ & 0.5 & $14(100 \%)$ & 0 & 0 \\
\hline \multirow[t]{4}{*}{ C. krusei $(n=1)$} & Fluconazole & 64 & & & 0 & 0 & $1(100 \%)$ \\
\hline & Voriconazole & 0.5 & & & $1(100 \%)$ & 0 & 0 \\
\hline & Itraconazole & 1 & & & NA & NA & NA \\
\hline & Amphotericin B & 1 & & & $1(100 \%)$ & 0 & 0 \\
\hline \multirow[t]{4}{*}{ Others $(n=6)$} & Fluconazole & $\leq 1-16$ & $\leq 2$ & 16 & NA & NA & NA \\
\hline & Voriconazole & $\leq 0.06-2$ & $\leq 0.06$ & 2 & NA & NA & NA \\
\hline & Itraconazole & $\leq 0.125-1$ & 0.125 & 1 & NA & NA & NA \\
\hline & Amphotericin B & $\leq 0.5-0.5$ & $\leq 0.5$ & 0.5 & NA & NA & NA \\
\hline
\end{tabular}

${ }^{a}$ Specific clinical breakpoints (CBPs) for Candida susceptibility to fluconazole and voriconazole were determined following CLSI [14], while CBPs for susceptibility

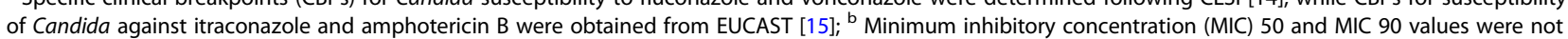
calculated for antifungal drugs against $C$. krusei owing to the small number of $C$. krusei case. Isolates of $C$. krusei are assumed to be intrinsically resistant to fluconazole. S, susceptible; I, intermediate; SDD, susceptible-dose dependent; R, resistant; NA, non-applicable;

identified several factors associated with 30-day mortality of surgical patients with candidemia, including ICU stay, recent abdominal surgery, recent cancer surgery, presence of CVC, renal failure, hypoalbuminemia, hyponatremia, thrombocytopenia, C. albicans infection and C. parapsilosis infection (Table 3). The results of the multivariable analysis for the risk factors associated with 30 -day mortality are presented in Table 4 . ICU stay (adjusted odds ratio (aOR) 6.55; 95\% confidence interval (CI) $1.85-23.22 ; \quad P=0.004)$, thrombocytopenia (aOR 5.72; CI 1.54-21.20; $P=0.009)$, and $C$. albicans infection (aOR 6.08; CI 1.53-24.19; $P=0.01$ ) were independent risk factors associated with increased 30-day mortality of surgical patients with candidemia.

\section{Discussion}

The present study focused on candidemia in surgical patients, and it showed that the proportion of surgical patients in the overall candidemia cases reached to $72.9 \%$, which was much higher than around 50-55\% reported in Europe, America, and Australia [4, 5, 9]. Moreover, our data also found the incidence of candidemia in surgical patients was 24.8 episodes per 1000 patient-days. This was dramatically higher compared with that observed in previous studies from North America and Europe, which has documented that the incidence density occurring in surgical ICU is nearly $0.6-0.98$ episodes per 1000 patient-days $[6,16]$. The variations in the incidence and the species distribution of candidemia may because the epidemiology of candidemia varied with geographic regions, type of hospital, patient populations, and study periods $[8,17]$.

Our study showed that the proportion of solid tumors was significantly higher in surgical patients than that in non-surgical patients. In solid-tumor patients with candidemia, several studies observed a predominance of $C$. albicans (around 41-53.7\%) and the variable proportion of C. parapsilosis, C. glabrata, and C. tropicalis, which 
Table 3 Univariable analysis of factors associated with 30-day mortality of surgical patients with candidemia

\begin{tabular}{|c|c|c|c|c|c|}
\hline Variable & Survivors $\boldsymbol{n}=139$ (80.8\%) & Deaths $\boldsymbol{n}=33$ (19.2\%) & Odds ratio & 95\% Confidence interval & $\boldsymbol{P}$ value \\
\hline Age & $61.6 \pm 14.4$ & $66.5 \pm 12.6$ & 1.03 & $1-1.06$ & 0.07 \\
\hline Age $\geq 65$ years & $57(41.0 \%)$ & $18(54.5 \%)$ & 1.73 & $0.8-3.71$ & 0.16 \\
\hline Male sex & $97(69.8 \%)$ & $22(66.7 \%)$ & 0.87 & $0.39-1.95$ & 0.73 \\
\hline \multicolumn{6}{|l|}{ Underlying diseases } \\
\hline Solid tumors & 77 (55.4\%) & $13(39.4 \%)$ & 0.52 & $0.24-1.14$ & 0.10 \\
\hline Diabetes mellitus & $24(17.3 \%)$ & $8(24.2 \%)$ & 1.53 & $0.62-3.81$ & 0.36 \\
\hline \multicolumn{6}{|l|}{ Predisposing factors } \\
\hline Current and former smokers & $42(30.2 \%)$ & $10(30.3 \%)$ & 1 & $0.44-2.29$ & 0.99 \\
\hline ICU stay & $38(27.3 \%)$ & $25(75.8 \%)$ & 8.31 & $3.45-20.01$ & $<0.001$ \\
\hline Recent abdominal surgery & $118(84.9 \%)$ & $23(69.7 \%)$ & 0.41 & $0.17-0.98$ & 0.046 \\
\hline Recent cancer surgery & $74(53.2 \%)$ & $11(33.3 \%)$ & 0.44 & $0.2-0.97$ & 0.04 \\
\hline Prior antibiotics exposure & $134(96.4 \%)$ & $32(97.0 \%)$ & 1.19 & $0.13-10.58$ & 0.87 \\
\hline Prior antifungal exposure & $20(14.4 \%)$ & $5(15.2 \%)$ & 1.06 & $0.37-3.08$ & 0.91 \\
\hline TPN & $130(93.5 \%)$ & $32(97.0 \%)$ & 2.22 & $0.27-18.13$ & 0.46 \\
\hline Presence of CVC & 49 (35.3\%) & $18(54.5 \%)$ & 2.2 & $1.02-4.75$ & 0.04 \\
\hline Mechanical ventilation & $15(10.8 \%)$ & $5(15.2 \%)$ & 1.48 & $0.5-4.4$ & 0.48 \\
\hline Presence of urinary catheter & $106(76.3 \%)$ & $21(63.6 \%)$ & 0.54 & $0.24-1.22$ & 0.14 \\
\hline Presence of gastric tube & 109 (78.4\%) & $23(69.7 \%)$ & 0.63 & $0.27-1.47$ & 0.29 \\
\hline \multicolumn{6}{|l|}{ Laboratory findings } \\
\hline Renal failure & $17(12.2 \%)$ & $14(42.4 \%)$ & 5.29 & $2.24-12.46$ & $<0.001$ \\
\hline Anemia & $116(83.5 \%)$ & $28(84.8 \%)$ & 1.11 & $0.39-3.18$ & 0.85 \\
\hline Hypoalbuminemia & $47(33.8 \%)$ & $24(72.7 \%)$ & 5.22 & $2.25-12.13$ & $<0.001$ \\
\hline Hyponatremia & $67(48.2 \%)$ & $6(18.2 \%)$ & 0.24 & $0.09-0.61$ & 0.003 \\
\hline Leukopenia & $14(10.1 \%)$ & $5(15.2 \%)$ & 1.59 & $0.53-4.79$ & 0.41 \\
\hline Thrombocytopenia & $10(7.2 \%)$ & $14(42.4 \%)$ & 9.51 & $3.7-24.42$ & $<0.001$ \\
\hline C. albicans infection & 39 (28.1\%) & $19(57.6 \%)$ & 3.48 & $1.59-7.62$ & 0.002 \\
\hline C. parapsilosis infection & 67 (48.2\%) & $7(21.2 \%)$ & 0.29 & $0.12-0.71$ & 0.007 \\
\hline C. glabrata infection & $15(10.8 \%)$ & $4(12.1 \%)$ & 1.14 & $0.35-3.69$ & 0.83 \\
\hline C. tropicalis infection & $11(7.9 \%)$ & $3(9.1 \%)$ & 1.16 & $0.31-4.43$ & 0.82 \\
\hline Fluconazole susceptibility & $103(74.1 \%)$ & $23(69.7 \%)$ & 0.8 & $0.35-1.85$ & 0.61 \\
\hline Empirical antifungal therapy & $94(67.6 \%)$ & $21(63.6 \%)$ & 0.84 & $0.38-1.85$ & 0.66 \\
\hline No antifungal therapy & $22(15.8 \%)$ & $5(15.2 \%)$ & 0.95 & $0.33-2.73$ & 0.92 \\
\hline \multicolumn{6}{|l|}{ Outcome } \\
\hline LOS (days) & $33(22-48)$ & $31(23-41.5)$ & 1 & $0.98-1.01$ & 0.52 \\
\hline
\end{tabular}

ICU, Intensive care unit; TPN, Total parenteral nutrition; CVC, central venous catheter; LOS, Length of hospital stay;

were 9.7, 20.2, and 7.1\% in France [18], 20.7, 19.2, and $7.6 \%$ in Spain [19], 15, 12, 20\% in Brazil [20], respectively. Nonetheless, we did not observe important differences regarding the distribution of Candida spp. between overall surgical patients and those with solid tumors. In the present study, the proportion of C. glabrata (11\%) and $C$. tropicalis (8.1\%) were relatively lower, whereas $C$. parapsilosis exceeded that $C$. albicans (33.7\%) to account for $43 \%$ of all isolates among surgical patients. In contrast to our findings, C. albicans was the most frequently isolated spp. from candidemia, and $C$. albicans and $C$. parapsilosis were observed with a frequency of 51 and $25 \%$ among surgical patients in Italy [12], 50.9 and $21.8 \%$ in surgical wards in Spain [11], and 48 and $7 \%$ among surgical ICU patients in the United States [6], respectively. Of note, we observed the frequency of $C$. parapsilosis isolates among surgical patients was higher than those among non-surgical patients. C. albicans with $39.1 \%$ was the predominant species isolated in non-surgical patients in our study, 
Table 4 Multivariable logistic regression analysis of risk factors for 30-day mortality of surgical patients with candidemia

\begin{tabular}{llll}
\hline Variable & Adjusted odds ratio & $95 \%$ Confidence interval & $P$ Value \\
\hline Age & 1.04 & $1.0-1.08$ & 0.06 \\
Solid tumors & 0.89 & $0.07-10.71$ & 0.93 \\
ICU stay & 6.55 & $1.85-23.22$ & 0.004 \\
Recent abdominal surgery & 0.33 & $0.09-1.24$ & 0.1 \\
Recent cancer surgery & 0.67 & $0.05-8.88$ & 0.76 \\
Presence of CVC & 0.98 & $0.28-3.38$ & 0.97 \\
Renal failure & 1.78 & $0.54-5.95$ & 0.35 \\
Hypoalbuminemia & 2.78 & $0.94-8.2$ & 0.06 \\
Hyponatremia & 0.35 & $0.1-1.24$ & 0.10 \\
Thrombocytopenia & 5.72 & $1.54-21.20$ & 0.009 \\
C.albicans infection & 6.08 & $1.53-24.19$ & 0.01 \\
C. parapsilosis infection & 1.07 & $0.24-4.7$ & 0.93 \\
\hline
\end{tabular}

$\mathrm{Cl}$, confidence interval; $\mathrm{ICU}$, Intensive care unit; CVC, central venous catheter;

followed by $C$. parapsilosis with $23.4 \%$, C. tropicalis with $20.3 \%$, and others. C. parapsilosis candidemia has been associated with CVC, TPN, prior antifungal therapy, neonates, and transplantation [17, 21-23]. Additionally, the transmission via the hands of health care workers, other medical devices and catheters may also be the risk factors for the nosocomial acquisition of $C$. parapsilosis infection [23]. The reasons for the high prevalence of candidemia due to $C$. parapsilosis among surgical patients are not completely understood, but the high use of invasive procedures and TPN in our study may partially explain the results. And thus, the optimal implementing device and catheter care may contribute to reduce episodes of $C$. parapsilosis BSI in our hospital. On the other hand, our findings showed the frequency of $C$. tropicalis candidemia was significantly lower in surgical patients than non-surgical patients. Moreover, C. tropicalis candidemia was found more frequently in surgical patients older than 65 years or with DM or in nonsurgical patients with hematological malignancies, which is similar to that reported in previous studies $[19,20,24,25]$.

In our series, the proportion of fluconazolesusceptible Candida isolates in surgical patients did not significantly differ from that observed in nonsurgical cases. Overall, on the basis of new speciesspecific CBPs, azole susceptibilities of Candida spp. were strikingly lower than the previously findings reported in the USA [26], parts of Italy and Spain [24], and Asian [27], but were similar to those observed in a prospective population-based surveillance for candidemia in Spain [4]. The elevated rates of fluconazoleSDD C. albicans, C. parapsilosis, and C. glabrata isolates emphasize the importance of the suitable and adequate systemic antifungal therapy for candidemia, as highly recommended in the guideline [28]. Our data confirmed that azole-resistant isolates were mainly observed in C. tropicalis and C. krusei, while amphotericin B had an excellent activity against all Candida spp. tested. As expected, the C. krusei isolate, which is intrinsically resistant to fluconazole, was demonstrated to be susceptible to voriconazole. Although fluconazole prophylaxis has been reported to reduce the incidence of invasive candidiasis in highrisk patients in the surgical ICU $[6,29]$, previous exposure to fluconazole in high-risk surgical patients was also observed to be associated with subsequent candidemia caused by fluconazole-resistant Candida isolates [30], in agreement with our results. Despite the absence of antifungal susceptibility testing of echinocandins in current study, many studies have reported low resistance to echinocandins in Candida isolates, except for $C$. parapsilosis $[4,24,26]$. Due to the good safety profile, few drug-drug interactions and good susceptibilities, echinocandins have been recommended as first-line agents to treat invasive candidiasis [28, 31]. However, considering high treatment costs of echinocandins and amphotericin B, and more serious adverse effects of the latter, fluconazole was the most commonly prescribed antifungal agents for primary treatment in our study. Indeed, the use of amphotericin B is limited due to its severe sideeffects in clinical practice, and thus echinocandins were commonly taken into consideration in the empirical antifungal treatment when candidemia may be caused by non-susceptible Candida isolates [28, 31]. After susceptibility results to antifungal drugs were reported, for fluconazole-susceptible isolates, if the patients were neither critical ill nor had the previous exposure to antifungal drugs, fluconazole was considered to be an alternative to an echinocandin. 
The association between prior surgery, especially prior abdominal surgery and a better outcome in candidemia patients has been observed in other studies $[10,11,32-34]$, which may be due to the lower severity of the underlying comorbidities, prompt antifungal therapy, and infection source control by CVC removal or appropriate surgical intervention [11, 33]. As mentioned previously, 30-day mortality of our surgical patients with candidemia were also lower than that of non-surgical patients. However, the difference did not reach statistical significance, probably because of similar percentages of ICU stay and $C$. albicans BSI between surgical and non-surgical patients, which were the risk factors for candidemia-related mortality in current study. The overall 30-day mortality in our surgical patients with candidemia was $19.2 \%$, which is similar to that reported in surgical wards and ICU in northeastern Italy [33]. It was relatively high for candidemia patients with recent surgery (including ICU) in central Italy [12] and those in surgical ICU in France [10], which was 38 and $45.2 \%$, respectively. In addition, the 30-day mortality of patients with candidemia hospitalized in surgical wards was observed to be $15.8 \%$ in a recent multicenter population study on candidemia in Spain [11], whereas it was as high as $38.7 \%$ in a retrospective study in Israel [35].

Not surprisingly, consistent with our data, ICU stay has been demonstrated to be independently associated with greater mortality in patients due to Candida BSI $[12,36]$, suggesting that severity of illness may affect the outcome of patients with candidemia. In agreement with previous reports $[37,38]$, we found that $C$. albicans was independently related to a poor outcome in surgical patients with candidemia, probably because of the relative virulence of $C$. albicans, patient's underlying diseases and host defense deficiencies [39]. Among surgical patients with candidemia, our multivariable analysis also revealed that thrombocytopenia was independently associated with 30-day mortality, as have been described in adult patients with candidemia by Jia et al [36]. Except for their key role in thrombosis and hemostasis, platelets play a crucial role in inflammation, immunity, and the infectious disease [40]. Actually, the responses of platelets to bacterial infections are complicated, and thus thrombocytopenia can be either harmful or protective in host defense against pathogens depending on the pathophysiological context [41]. In any case, thrombocytopenia remained correlated to mortality of sepsis patients [40]. The platelet decrease in sepsis may be due to reduced thrombopoiesis, hemodilution, sequestration, the depletion of platelets, and immunemediated destruction of platelets [40]. However, the mechanisms for platelet decrease and platelet function in candidemia have not been completely elucidated, and further studies are necessary.

There were several limitations to our study. First, due to the limitations of a retrospective cohort design, some variables cannot be analyzed. Second, echinocandin susceptibilities were not assessed since their minimum inhibitory concentration (MIC) CBPs were not interpreted by the CLSI or EUCAST at the time. And echinocandin regimens were empirically administered according to the CLSI or EUCAST document. Furthermore, the present study was performed at three hospital campuses and the results may not be applicable to other settings.

\section{Conclusions}

In conclusion, our study showed that $C$. parapsilosis was the most common species surpassing $C$. albicans, suggesting the high prevalence of non-C. albicans species in surgical patients with candidemia. Moreover, among patients with recent surgery, risk factors for candidemia caused by various Candida species and resistance of these isolates to azoles, particularly fluconazole, should be strongly considered when starting an empirical treatment. The recognition of prognostic factors for candidemia in surgical patients may help clinicians to assess and prevent poor outcomes.

\section{Abbreviations}

BSI: Bloodstream infection; CVC: Central venous catheter; ICU: Intensive care unit; CBP: Clinical breakpoints; CLSI: Clinical Laboratory Standards Institute; EUCAST: European Committee on Antimicrobial Susceptibility Testing; MIC: Minimum inhibitory concentration; OR: Odds ratios; Cl: Confidence intervals; DM: Diabetes mellitus; LOS: Length of hospital stay; TPN: Total parenteral nutrition; SDD: Susceptible dose dependent

\section{Acknowledgements}

We are grateful to Dr. Liqiang Zheng at the Department of Clinical Epidemiology, Shengjing Hospital, for his valuable advice on statistical analysis.

\section{Authors' contributions}

WZ contributed to study design, data analysis, drafting and revising of the manuscript. XS and HW contributed to this work by data acquisition and revising of the manuscript. RZ had a contribution in study conception and design, and revising of the manuscript. All co-authors have given the final approval of the version to be published.

\section{Funding}

RZ is supported by 345 Talent Project of Shengjing Hospital. This funding source was involved in data collection and data analysis.

\section{Availability of data and materials}

The datasets used in this study are available from the corresponding author upon reasonable request.

\section{Ethics approval and consent to participate}

The study was approved by the Medical Ethics Committee in Shengjing Hospital of China Medical University (reference number 2019PS636K). Informed consent was not obtained from individual patients because the data were collected retrospectively. 


\section{Competing interests}

The authors declare no competing interests. The authors alone are responsible for the content and the writing of the paper.

\section{Author details}

'Department of Pulmonary and Critical Care Medicine, Shengjing Hospital of China Medical University, No. 36 Sanhao Street, Heping District, Shenyang 110004, Liaoning, China. ${ }^{2}$ Department of Pulmonary and Critical Care Medicine, Anshan Central Hospital, No. 77 Nanzhonghua Street, Tiedong District, Anshan 114000, Liaoning, China.

Received: 25 February 2020 Accepted: 7 July 2020 Published online: 13 July 2020

\section{References}

1. Wisplinghoff H, Bischoff T, Tallent SM, Seifert H, Wenzel RP, Edmond MB. Nosocomial bloodstream infections in US hospitals: analysis of 24,179 cases from a prospective nationwide surveillance study. Clin Infect Dis. 2004;39: 309-17.

2. Magill SS, Edwards JR, Bamberg W, Beldavs ZG, Dumyati G, Kainer MA, et al. Multistate point-prevalence survey of health care-associated infections. N Engl J Med. 2014;370:1198-208.

3. Hassan I, Powell G, Sidhu M, Hart WM, Denning DW. Excess mortality, length of stay and cost attributable to candidaemia. J Inf Secur. 2009;59:360-5.

4. Puig-Asensio M, Padilla B, Garnacho-Montero J, Zaragoza O, Aguado JM, Zaragoza $\mathrm{R}$, et al. Epidemiology and predictive factors for early and late mortality in Candida bloodstream infections: a population-based surveillance in Spain. Clin Microbiol Infect. 2014;20:0245-54.

5. Das I, Nightingale P, Patel M, Jumaa P. Epidemiology, clinical characteristics, and outcome of candidemia: experience in a tertiary referral center in the UK. Int J Infect Dis. 2011;15:e759-63.

6. Blumberg HM, Jarvis WR, Soucie JM, Edwards JE, Patterson JE, Pfaller MA et al. Risk factors for candidal bloodstream infections in surgical intensive care unit patients: the NEMIS prospective multicenter study. The National Epidemiology of mycosis survey. Clin Infect Dis. 2001;33:177-86.

7. Vaezi A, Fakhim H, Khodavaisy S, Alizadeh A, Nazeri M, Soleimani A, et al. Epidemiological and mycological characteristics of candidemia in Iran: a systematic review and meta-analysis. J Mycol Med. 2017;27:146-52.

8. Diekema D, Arbefeville S, Boyken L, Kroeger J, Pfaller M. The changing epidemiology of healthcare-associated candidemia over three decades. Diagn Microbiol Infect Dis. 2012;73:45-8.

9. Arendrup MC. Candida and candidaemia. Susceptibility and epidemiology Dan Med J. 2013;60:B4698.

10. Charles PE, Doise JM, Quenot JP, Aube H, Dalle F, Chavanet P, et al. Candidemia in critically ill patients: difference of outcome between medical and surgical patients. Intensive Care Med. 2003;29:2162-9.

11. Vena A, Bouza E, Valerio M, Padilla B, Pano-Pardo JR, Fernandez-Ruiz M, et al. Candidemia in non-ICU surgical wards: comparison with medical wards. PLoS One. 2017;12:e0185339.

12. Orsetti E, Brescini L, Mazzanti S, Trave F, Morroni G, Masucci A, et al. Characterisation of candidemia in patients with recent surgery: a 7-year experience. Mycoses. 2019;62:1056-63.

13. Zhang W, Song X, Wu H, Zheng R. Epidemiology, risk factors and outcomes of Candida albicans vs. non-albicans candidaemia in adult patients in Northeast China. Epidemiol Infect. 2019;147:e277.

14. CLSI. Performance Standards for Antifungal Susceptibility Testing of Yeasts, 1st Edition. CLSI supplement M60. Wayne: Clinical and Laboratory Standards Institute. [https://clsi.org/standards/products/microbiology/documents/m60/ ] Accessed 12 June 2019.

15. EUCAST. European Committee on Antimicrobial Susceptibility Testing Antifungal Agents Breakpoint tables for interpretation of MICs, Version 9.0, valid from 2018-02-12. [http://www.eucast.org/clinical_breakpoints/] Accessed 12 June 2019.

16. Bougnoux ME, Kac G, Aegerter P, d'Enfert C, Fagon JY, CandiRea SG Candidemia and candiduria in critically ill patients admitted to intensive care units in France: incidence, molecular diversity, management and outcome. Intensive Care Med. 2008;34:292-9.

17. Guinea J. Global trends in the distribution of Candida species causing candidemia. Clin Microbiol Infect. 2014;20(Suppl 6):5-10.
18. Lortholary O, Renaudat C, Sitbon K, Desnos-Ollivier M, Bretagne S, Dromer F, et al. The risk and clinical outcome of candidemia depending on underlying malignancy. Intensive Care Med. 2017;43:652-62.

19. Puig-Asensio M, Ruiz-Camps I, Fernandez-Ruiz M, Aguado JM, Munoz P, Valerio $\mathrm{M}$, et al. Epidemiology and outcome of candidaemia in patients with oncological and haematological malignancies: results from a populationbased surveillance in Spain. Clin Microbiol Infect. 2015;21:491 e1-10.

20. Bergamasco MD, Garnica M, Colombo AL, Nucci M. Epidemiology of candidemia in patients with hematologic malignancies and solid tumours in Brazil. Mycoses. 2013;56:256-63.

21. Almirante B, Rodriguez D, Cuenca-Estrella M, Almela M, Sanchez F, Ayats J, et al. Epidemiology, risk factors, and prognosis of Candida parapsilosis bloodstream infections: case-control population-based surveillance study of patients in Barcelona, Spain, from 2002 to 2003. J Clin Microbiol. 2006;44: 1681-5.

22. Levin AS, Costa SF, Mussi NS, Basso M, Sinto SI, Machado C, et al. Candida parapsilosis fungemia associated with implantable and semi-implantable central venous catheters and the hands of healthcare workers. Diagn Microbiol Infect Dis. 1998;30:243-9.

23. Trofa D, Gacser A, Nosanchuk JD. Candida parapsilosis, an emerging fungal pathogen. Clin Microbiol Rev. 2008;21:606-25.

24. Bassetti M, Merelli M, Righi E, Diaz-Martin A, Rosello EM, Luzzati R, et al. Epidemiology, species distribution, antifungal susceptibility, and outcome of candidemia across five sites in Italy and Spain. J Clin Microbiol. 2013;51: 4167-72.

25. Nucci M, Colombo AL. Candidemia due to Candida tropicalis: clinical, epidemiologic, and microbiologic characteristics of 188 episodes occurring in tertiary care hospitals. Diagn Microbiol Infect Dis. 2007;58:77-82.

26. Lyon GM, Karatela S, Sunay S, Adiri Y. Antifungal susceptibility testing of Candida isolates from the Candida surveillance study. J Clin Microbiol. 2010; 48:1270-5.

27. Wang H, Xu YC, Hsueh PR. Epidemiology of candidemia and antifungal susceptibility in invasive Candida species in the Asia-Pacific region. Future Microbiol. 2016;11:1461-77.

28. Pappas PG, Kauffman CA, Andes DR, Clancy CJ, Marr KA, Ostrosky-Zeichner L, et al. Clinical practice guideline for the Management of Candidiasis: 2016 update by the Infectious Diseases Society of America. Clin Infect Dis. 2016; 62:e1-50.

29. Pelz RK, Hendrix CW, Swoboda SM, Diener-West M, Merz WG, Hammond J, et al. Double-blind placebo-controlled trial of fluconazole to prevent candidal infections in critically ill surgical patients. Ann Surg. 2001;233:5428.

30. Zilberberg M, Yu HT, Chaudhari P, Emons MF, Khandelwal N, Shorr AF. Relationship of fluconazole prophylaxis with fungal microbiology in hospitalized intra-abdominal surgery patients: a descriptive cohort study. Critical care [London, England). 2014;18:590.

31. Cornely OA, Bassetti M, Calandra T, Garbino J, Kullberg BJ, Lortholary O, et al. ESCMID* guideline for the diagnosis and management of Candida diseases 2012: non-neutropenic adult patients. Clin Microbiol Infect. 2012; 18(Suppl 7):19-37.

32. Wang TY, Hung CY, Shie SS, Chou PC, Kuo CH, Chung FT, et al. The clinical outcomes and predictive factors for in-hospital mortality in nonneutropenic patients with candidemia. Medicine. 2016;95:e3834.

33. Luzzati R, Merelli M, Ansaldi F, Rosin C, Azzini A, Cavinato S, et al. Nosocomial candidemia in patients admitted to medicine wards compared to other wards: a multicentre study. Infection. 2016;44:747-55.

34. Lausch KR, Sogaard M, Rosenvinge FS, Johansen HK, Boysen T, Roder B, et al. High incidence of candidaemia in a nationwide cohort: underlying diseases, risk factors and mortality. Int J Infect Dis. 2018;76:58-63.

35. Eliakim-Raz N, Babaoff R, Yahav D, Yanai S, Shaked H, Bishara J. Epidemiology, microbiology, clinical characteristics, and outcomes of candidemia in internal medicine wards-a retrospective study. Int J Infect Dis. 2016:52:49-54.

36. Jia X, Li C, Cao J, Wu X, Zhang L. Clinical characteristics and predictors of mortality in patients with candidemia: a six-year retrospective study. Eur J Clin Microbiol Infect Dis. 2018;37:1717-24.

37. Wang H, Wu DW, Han H, Yue JF, Zhang F, Shan TC, et al. Antibiotics exposure, risk factors, and outcomes with Candida albicans and nonCandida albicans candidemia. Results from a multi-center study. Saudi Med J. 2014;35:153-8. 
38. Weinberger M, Leibovici L, Perez S, Samra Z, Ostfeld I, Levi I, et al. Characteristics of candidaemia with Candida-albicans compared with nonalbicans Candida species and predictors of mortality. The Journal of hospital infection. 2005;61:146-54.

39. Krcmery V, Barnes AJ. Non-albicans Candida spp. causing fungaemia: pathogenicity and antifungal resistance. The Journal of hospital infection. 2002:50:243-60

40. Vardon-Bounes F, Ruiz S, Gratacap MP, Garcia C, Payrastre B, Minville V. Platelets are critical key players in Sepsis. Int J Mol Sci. 2019;20:3494.

41. Nicolai L, Gaertner F, Massberg S. Platelets in host defense: experimental and clinical insights. Trends Immunol. 2019;40:922-38.

\section{Publisher's Note}

Springer Nature remains neutral with regard to jurisdictional claims in published maps and institutional affiliations.

Ready to submit your research? Choose BMC and benefit from:

- fast, convenient online submission

- thorough peer review by experienced researchers in your field

- rapid publication on acceptance

- support for research data, including large and complex data types

- gold Open Access which fosters wider collaboration and increased citations

- maximum visibility for your research: over $100 \mathrm{M}$ website views per year

At $\mathrm{BMC}$, research is always in progress.

Learn more biomedcentral.com/submissions 